\title{
Clinical and Functional Characteristics of a Novel KLF11 Cys354Phe Variant Involved in Maturity-Onset Diabetes of the Young
}

\author{
Yujing Sun $\mathbb{D}^{1,2,3}$ Jingru Qu $\mathbb{D}^{1,2,3}$ Jing Wang, ${ }^{1,2,3}$ Ruxing Zhao, ${ }^{1,2,3}$ Chuan Wang $\mathbb{D}^{1,2,3}$ \\ Li Chen $\mathbb{D}^{1,2,3}$ and Xinguo Hou $\mathbb{D i}^{1,2,3}$
}

${ }^{1}$ Department of Endocrinology, Qilu Hospital, Cheeloo College of Medicine, Shandong University, Jinan 250012, China

${ }^{2}$ Institute of Endocrine and Metabolic Diseases of Shandong University, Jinan, 250012 Shandong Province, China

${ }^{3}$ Jinan Clinical Research Center for Endocrine and Metabolic Diseases, Jinan, 250012 Shandong Province, China

Correspondence should be addressed to Li Chen; chenli3@medmail.com.cn and Xinguo Hou; houxinguo@sdu.edu.cn

Received 31 July 2020; Revised 18 November 2020; Accepted 10 January 2021; Published 1 February 2021

Academic Editor: Xiaomu Kong

Copyright () 2021 Yujing Sun et al. This is an open access article distributed under the Creative Commons Attribution License, which permits unrestricted use, distribution, and reproduction in any medium, provided the original work is properly cited.

\begin{abstract}
Background. Mutations in human KLF11 may lead to the development of maturity-onset diabetes of the young 7 (MODY7). This occurs due to impaired insulin synthesis in the pancreas. To date, the clinical and functional characteristics of the novel KLF11 mutation c.1061G > T have not yet been reported. Methods. Whole-exon sequencing was used to screen the proband and family members with clinical suspicion of the KLF11 variant. Luciferase reporter assays were used to investigate whether the KLF11 variant binds to the insulin promoter. Real-time PCR, western blotting, and glucose-stimulated insulin secretion (GSIS) analysis were used to analyze the KLF11 variant that regulates insulin expression and insulin secretion activity in beta cell lines. The Freestyle Libre H (Abbott Diabetes Care Ltd) was used to dynamically monitor the proband daily blood glucose levels. Results. Mutation screening for the whole exon genes identified a heterozygous $K L F 11$ (c.1061G > T) variant in the proband, her mother, and her maternal grandfather. Cell-based luciferase reporter assays using wild-type and mutant transgenes revealed that the KLF11 (c.1061G > T) variant had impaired insulin promoter regulation activity. Moreover, this variant was found to impair insulin expression and insulin secretion in pancreatic beta cells. The proband had better blood glucose control without staple food intake $(p<0.05)$. Conclusions. Herein, for the first time, we report a novel KLF11 (c.1061G>T) monogenic mutation associated with MODY7. This variant has impaired insulin promoter regulation activity and impairs insulin expression and secretion in pancreatic beta cells. Therefore, administering oral antidiabetic drugs along with dietary intervention may benefit the proband.
\end{abstract}

\section{Introduction}

KLF11 was first cloned in a human cystic fibrosis pancreatic adenocarcinoma cell line by Urrutia et al. in 1998 [1]. KLF11 is a pancreas-enriched transcription factor that has elicited significant attention from researchers due to its role as a negative regulator of exocrine cell growth both in vitro and in vivo [2]. Previous studies have shown that KLF11 is involved in the pathophysiological process of diabetes development [3-5]. Moreover, mutations in human KLF11 may lead to the development of maturity-onset diabetes of the young 7 (MODY7). Neve et al. were the first to study
MODY7 [2]. In 2005, genetic screening on two probands with a family history of early onset type 2 diabetes revealed that one proband had type 2 diabetes, while the other had reduced glucose tolerance [2]. This is due to the impairment of insulin synthesis from the pancreas. Genetic analysis of KLF11 has revealed two rare variants (Ala347Ser and Thr220Met) that are segregated in families with early onset type 2 diabetes and significantly impair its transcriptional activity [2].

In 2019, a novel KLF11 variant (p.His418Gln) was reported; this was associated with early childhood-onset type $1 \mathrm{~B}$ diabetes [6]. As such, KLF11 is a valid candidate gene to 
determine the genetic predisposition to early onset and type 2 diabetes, as defects in this gene may lead to early onset or polygenic type 2 diabetes [7]. In addition to KLF11, there may be other unknown factors that need further exploration. However, KLF11, due to its role as a MODY gene, is a potential therapeutic target for adult-onset diabetes.

In this study, we report a novel KLF11 variant associated with MODY7 and explore its clinical features, possible pathogenesis, optimal treatment, and functional characteristics. Furthermore, we also investigated whether dietary intervention may benefit diabetic patients with KLF11 mutation.

\section{Materials and Methods}

\subsection{Patients}

2.1.1. Proband. The proband (III-4, Figure 1) was a female subject aged 23 years, with normal development and moderate nutritional status. However, one physical examination on September 2nd, 2018, revealed that the proband's fasting blood glucose level was $13.9 \mathrm{mmol} / \mathrm{L}$. Immediately prior to admission to the local hospital, the proband's fasting blood glucose level was $10.36 \mathrm{mmol} / \mathrm{L}$, and $\mathrm{HbAlc}$ levels were $11.5 \%$. Due to the high blood glucose level, the doctor treated her with insulin and oral hypoglycemic agents. One month later, the proband's blood glucose control worsened after gradually adjusting the treatment to saxagliptin $(2.5 \mathrm{mg} /$ day $)$ and voglibose $(0.6 \mathrm{mg} /$ day $)$, which was followed by admission to the QiLu Hospital for further treatment. The patient reported no polydipsia, polyuria, weight loss, or blurred vision and no numbness of the limbs, fatigue, or discomfort during this time.

All procedures performed in the study involving human participants were in accordance with the ethical standards of the institutional and/or national research committee and with the 1964 Helsinki Declaration and its later amendments or comparable ethical standards. This study was approved by the ethics committee of the QiLu Hospital of Shandong University (No: KYLL-2020(KS)-069). All the subjects included in our study voluntarily signed an informed consent form, which was reviewed by the ethical committee.

2.1.2. Family History. There was no history of diabetes on the paternal side of the family, whereas there was one diabetic patient among maternal relatives (maternal grandfather).

2.1.3. Physical Examination. The proband's height was $168.5 \mathrm{~cm}$; she weighed $54 \mathrm{~kg}$ and had a body mass index (BMI) of $19.02 \mathrm{~kg} / \mathrm{m}^{2}$. Moreover, the proband did not exhibit any obvious abnormalities of the heart, lungs, or abdomen, and there was no edema in either of the lower limbs.

2.1.4. Treatment Methods. Due to the unsatisfactory control of both fasting and postprandial blood glucose levels, the treatment was changed to insulin glargine injections (10 units at bedtime), oral saxagliptin ( $2.5 \mathrm{mg}$; once/day), and oral voglibose ( $0.2 \mathrm{mg}$; thrice/day).

2.1.5. Clinical Data Collection. The subject's family provided informed consent and was enrolled in September 2018. We collected comprehensive clinical data, such as results of physical examinations, medical history, pedigree, and levels of related metabolism products.

2.2. Mutation Analysis. Peripheral blood was collected from four family members (the proband, her parents, and her maternal grandfather). The EDTA anticoagulation and E.Z.N.A. ${ }^{\circledR}$ Blood DNA Mini Kit (omega Bio-Tek, Inc. D3392) were used to extract the genomic DNA from peripheral blood leukocytes, which was then sent to Beijing Fujun Gene Biotechnology Co., Ltd. for whole-exome sequencing.

2.3. In Silico Analysis of the KLF11 Variant. Using the phyre2 server, the three-dimensional (3D) structures of wild-type KLF11 (WT-KLF11) and its variant (Cys354Phe-KLF11) were predicted using the threading method and comprehensively analyzed through head-to-head comparison of the final models. The WT-KLF11 and Cys354Phe-KLF11 3D structural models were established as reference models [8]. Structure visualization was performed using the SAVES v5.0 server.

2.4. Plasmid Information. The plasmid vector encoding the insulin promoter sequence pGL3-basic-INS, which was first reported by Bernadette Neve et al. [2], was synthesized by Biosune Biotechnology (Shanghai) Co., Ltd. The pCDNA3.1-WT-KLF11 plasmid was purchased from Biosune Biotechnology (Shanghai) Co., Ltd. The pCDNA3.1C354F-KLF11 plasmid was constructed using the QuikChange site-directed mutagenesis kit (Agilent Technologies, Inc., Santa Clara, CA), according to the manufacturer's instructions. All constructs were verified by sequencing on an ABI 3730xl sequencer. The construct was completely sequenced and used as the template in other cloning designs.

2.5. Western Blotting. Human embryonic kidney (HEK) 293 cells were cotransfected with plasmids encoding Cys354Phe-KLF11 and WT-KLF11 and were cultured as previously described [9]. At $48 \mathrm{~h}$ posttransfection, the cells were harvested and subjected to sodium dodecyl sulfatepolyacrylamide gel electrophoresis (SDS-PAGE). Western blotting was performed using a mouse monoclonal antiKLF11 primary antibody (Origene, Cat: TA811001s, $1: 1000)$ and horseradish peroxidase-conjugated goat antimouse IgG polyclonal secondary antibody (Zhongshan Golden Bridge, Cat: ZB-2305, 1 : 10000). Protein bands were detected using a chemiluminescence kit (Millipore, CA, USA, WBKLS0050) and imaged using a chemiluminescence imaging system (Shanghai Qinxiang Scientific Instrument Co., Ltd.).

2.6. Luciferase Reporter Assay. HEK 293 cells were seeded in 96-well culture plates $(10,000$ cells/well in $200 \mu \mathrm{L}$ of culture media) and cultured for $24 \mathrm{~h}$. Then, each cell group was transfected separately with a pRL-TK plasmid, pGL3-basicINS, and pCDNA3.1-WT-KLF11 or pCDNA3.1-C354FKLF11 using LipofectamineTM 2000(Invitrogen, cat:11668027), according to the manufacturer's instructions. At $48 \mathrm{~h}$ posttransfection, the dual-luciferase reporter assay was 

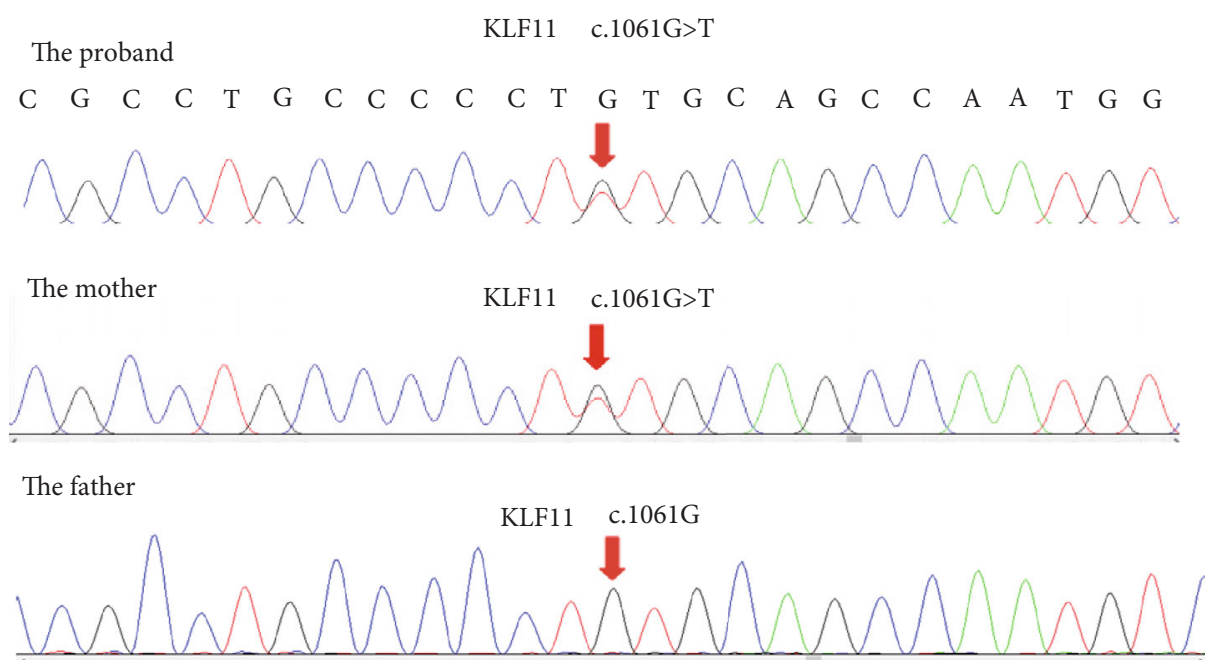

(a)

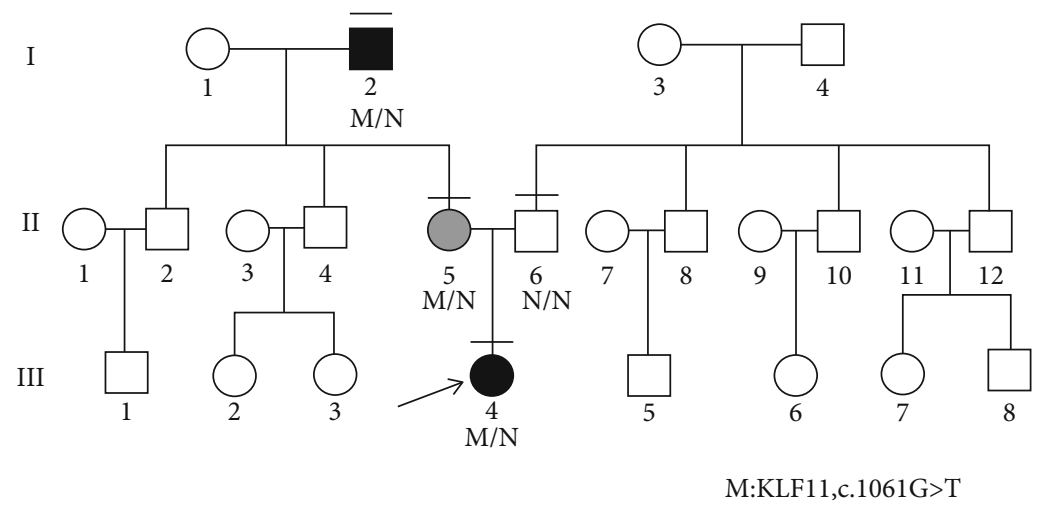

(b)

Figure 1: Partial sequence diagram of KLF11 and the pedigree of the studied family members. (a) Partial sequence diagram of KLF11. A heterozygous c.1061G > T transition mutation, causing the substitution of cysteine by phenylalanine at codon 354 is shown using an arrow (GenBank accession number: NM_003597.4). (b) The pedigree of the study family. Women are represented using circles and men, using squares. The black symbols indicate individuals with diabetes. The grey symbol indicates individuals with prediabetes. The proband is denoted by an arrow. The horizontal lines indicate individuals who underwent molecular analysis. The p.Cys354Phe variant of KLF11 was identified in I-2, II-5, and III-4. The N symbols denote the people that carry the WT gene, and the M symbols denote the people that carry the Cys354Phe variant.

performed using the Luciferase Reporter Assay Kit (Promega, E1910).

2.7. Insulin Secretion and Insulin Content Assay. INS1 cells were cotransfected with plasmids encoding Cys354PheKLF11 and WT-KLF11 to investigate insulin secretion and insulin contents, as described previously [10]. Insulin secretion was determined using a static incubation method under conditions of $5 \% \mathrm{CO}_{2}$ and $95 \%$ air at $37^{\circ} \mathrm{C}$, as previously described $[11,12]$. Cells were seeded at a density of $2 \times 10^{5}$ cells in 24-well plates and cultured in $1 \mathrm{~mL}$ of Dulbecco's Modified Eagle Medium (DMEM, $25 \mathrm{mmol} / \mathrm{L}$ glucose). After $48 \mathrm{~h}$, the medium was removed, and cells were washed once with HEPES-balanced Krebs Ringer Bicarbonate Buffer (119 mmol/L NaCl, $4.74 \mathrm{mmol} / \mathrm{L} \mathrm{KCl,} 2.54 \mathrm{mmol} / \mathrm{L} \mathrm{CaCl}_{2}$, $7.4 \mathrm{mmol} / \mathrm{L} \quad \mathrm{MgCl}_{2}, \quad 1.19 \mathrm{mmol} / \mathrm{L} \quad \mathrm{KHPO}_{4}, \quad 25 \mathrm{mmol} / \mathrm{L}$ $\mathrm{NaHCO}_{3}, 10 \mathrm{mmol} / \mathrm{L}$ HEPES, pH 7.4) containing $0.5 \%$ bovine serum albumin (BSA) without glucose. Next, the cells were preincubated in HEPES-balanced Krebs-Ringer Bicar- bonate Buffer with $0.5 \% \mathrm{BSA}$ and $5 \mathrm{mmol} / \mathrm{L}$ glucose for 30 min. After washing twice with HEPES-balanced KrebsRinger Bicarbonate Buffer, INS1 cells were incubated in HEPES-balanced Krebs-Ringer Bicarbonate Buffer supplemented with $0.5 \%$ BSA and varying concentrations of glucose. The media were then collected and assayed for immunoreactive insulin via an enzyme-linked immunosorbent assay (ELISA), with mouse insulin being used as a standard. A volume of $200 \mu \mathrm{L}$ of $1 \mathrm{~mol} / \mathrm{L} \mathrm{NaOH}$ was added to each well to solubilize the cells in order to determine the cellular protein contents using an ELISA assay kit (Millipore, EZRMI-13 K). For the measurement of cellular insulin content, $1 \mathrm{~mL}$ of acid ethanol was added to the wells, which were then sealed with a pressure-sensitive film. The extract was collected after $24 \mathrm{~h}$ incubation at $4^{\circ} \mathrm{C}$ and was then diluted and assayed by ELISA.

2.8. Real-Time PCR. INS1 cells were isolated using the TRIzol reagent (Takara, T9108). The cDNA was generated using the 
HiScript Q RT SuperMix for qPCR (Takara, DRR047S), and the real-time PCR assays were conducted with an LC480 Light Cycler using the following primer sequences: Ins1 forward primer, GAAGAGGCCATCAAGCAGATCACT; Ins1 reverse primer, ATTGTTCCACAATGCCACGCT; GAPDH forward primer, GCCTTCCGTGTTCCTACC; and GAPDH reverse primer, GCCTGCTTCACCACCTTC. Relative gene expression was determined using a comparative method $(2$ $\triangle \triangle \mathrm{CT})$. GAPDH was used as an internal standard.

2.9. Statistical Analysis. All data are presented as the means \pm standard deviations (SDs) or means \pm standard errors of the means (SEMs). Statistical comparisons were performed by using two-tailed, paired Student's $t$-test for datasets involving only two groups, or by using one-way ANOVA in the case of data involving more than two groups. Then, the Dunnett's and Bonferroni's post hoc tests were performed for multiple comparisons. All tests were performed using GraphPad Prism 8. Every experiment was repeated at least thrice independently. Representative experimental results are shown in the figures. A $p$ value of $<0.05$ was considered statistically significant.

\section{Results}

3.1. Clinical Manifestation. As the proband was first diagnosed with diabetes at the age of 23 and treated with insulin and oral hypoglycemic agents, it was crucial to identify which type of diabetes the proband had. After three weeks of treatment, the HbA1c levels were 6.6\%. Therefore, this treatment plan was maintained for one and a half years, and the patient's blood glucose level was controlled and stable during this time.

3.2. Laboratory Data. Islet autoantibody screening revealed an absence of glutamic acid decarboxylase (GAD), tyrosine phosphatase antibodies (IA-1ABs), anti-insulin cell antibodies (ICA-IgG), insulin autoantibodies (IAAs), and $\beta$-cell-specific zinc transporter 8 . When the proband's blood glucose level was normal and stable, an oral glucose tolerance test (OGTT) was performed simultaneously with insulin and Cpeptide release experiments to assess islet function (Table 1). As the maternal grandfather of the proband was a diabetes patient, we performed genetic testing for the proband. As such, we identified a heterozygous variant of KLF11 (c.1061G > T, p.Cys354Phe) via whole-exome sequencing. Moreover, the proband exhibited no abnormalities in the thyroid function, hepatic and renal function, blood lipid profile, or urine microalbumin.

3.3. Clinical Characteristics of Family Members and Their Genetic Testing Results. The maternal grandfather of the proband (I-2) was diagnosed with type 2 diabetes at 66 years of age and treated with oral hypoglycemic agents. His blood HbA1c level was $6.0 \%$ during his last examination (Table 2). The parents of the proband were never diagnosed with diabetes, but the mother (II-5) was found to have a fasting blood glucose level of $5.8 \mathrm{mmol} / \mathrm{L}$ at the physical examination. Genetic testing for MODY facilitates a correct diagnosis, thereby enabling treatment optimization and
TABLE 1: Blood glucose, insulin, and C-peptide levels of the proband and her mother.

\begin{tabular}{lcccccc}
\hline & \multicolumn{2}{c}{$\begin{array}{c}\text { Blood glucose } \\
\text { Time }(\mathrm{h})\end{array}$} & \multicolumn{2}{c}{ Insulin } & \multicolumn{2}{c}{ C-peptide } \\
& II-5 & III-4 & II-5 & III-4 & II-5 & III-4 \\
\hline 0 & 5.27 & 6.98 & 3.12 & 8.99 & 0.66 & 0.31 \\
$0.5 \mathrm{~h}$ & 10.47 & 9.02 & 24.1 & 11.27 & 3.97 & 0.54 \\
$1 \mathrm{~h}$ & 10.91 & 10.3 & 33.38 & 15.1 & 5.63 & 0.95 \\
$2 \mathrm{~h}$ & 6.57 & 12.43 & 59.63 & 17.45 & 6.37 & 1.44 \\
$3 \mathrm{~h}$ & 3.38 & 9.81 & 29.38 & 15.2 & 3.07 & 1.49 \\
\hline
\end{tabular}

allowing the monitoring of asymptomatic family members. Therefore, genetic tests were conducted for the parents and maternal grandfather of the proband. As expected, the heterozygous variant of KLF11 (c.1061G > T, p.Cys354Phe) was also identified in the mother and maternal grandfather (Figure 1(a)). In order to confirm whether the mother was an asymptomatic member, we performed an OGTT simultaneously with insulin and C-peptide release experiments (Table 1). Our results showed that the mother of the proband can be diagnosed with impaired glucose tolerance, which means she is prediabetic and may develop diabetes in the future.

As there are differences in the clinical phenotypes caused by the same mutation even within the same family, these results may indicate that KLF11 mutations are associated with incomplete penetrance. In summary, our results indicate that the KLF11 (c.1061G > T) variant is associated with diabetes in this family.

3.4. Sequencing Results and Biochemical Characterization of the KLF11 (c.1061G>T) Variant. The conserved domain of human KLF11 consists of an extracellular region that comprises three transcriptional repressor domains (TRD) and a zinc finger domain. KLF11, as a member of the Sp1/KLF family, has been predicted to bind to either GC-rich or CACC sequences. The Cys354Phe-KLF11 variant was mapped to a novel hydrophobic glycine-glutamine-proline-rich domain that was observed in the corresponding region of its fly ortholog, cabut (Figure 2(a)). The KLF11 Cys354Phe variant was also mapped to the previously characterized transcriptional regulatory domain 3 (TRD3). Multiple amino acid sequence alignments using Clustal W showed that Cys354Phe-KLF11 was conserved across various species (Figure 2(b)). It was predicted that mutations in this buried site led to its exposure on the surface of the protein, thereby modifying the protein activity. In addition, due to the exposure of this site at the surface of the protein after mutation, it might be located in a larger transcriptional blocking domain and affect some transcriptional functions (Figure 2(c)).

3.5. Functional Characterization of the KLF11 (c.1061G > T) Variant. The link between the KLF11 (c.1061G > T) variant and the putative diabetes pathophysiological process was assessed. The KLF11 (c.1061G > T) gene variant did not affect protein expression levels (Figures 3(a) and 3(b)). The results 
TABLe 2: Clinical characteristics of the study family.

\begin{tabular}{|c|c|c|c|c|}
\hline & Proband & Maternal grandfather & Mother $\ddagger$ & Father \\
\hline Birth weight (kg) & 3.15 & No data & No data & $\begin{array}{l}\text { No } \\
\text { data }\end{array}$ \\
\hline \multicolumn{5}{|l|}{ At diagnosis } \\
\hline Age (yr) & 23 & 66 & - & - \\
\hline Height $(\mathrm{cm})$ & 168.5 & 172 & - & - \\
\hline Body weight (kg) & 54 & 65 & - & - \\
\hline BMI $\left(\mathrm{kg} / \mathrm{m}^{2}\right)$ & 19.02 & 21.97 & - & - \\
\hline $\mathrm{DKA} \dagger$ & No & No & - & - \\
\hline $\begin{array}{l}\text { Blood glucose } \\
(\mathrm{mmol} / \mathrm{L})\end{array}$ & 13.9 & No data & - & - \\
\hline \%) & 11.5 & No data & - & - \\
\hline \multicolumn{5}{|c|}{ At the latest examination } \\
\hline Age (yr) & 24 & 70 & 50 & 50 \\
\hline Height $(\mathrm{cm})$ & 168.5 & 172 & 163 & 173 \\
\hline Body weight (kg) & 50 & 55 & 50 & 65 \\
\hline BMI $\left(\mathrm{kg} / \mathrm{m}^{2}\right)$ & 17.61 & 18.59 & 18.82 & 21.72 \\
\hline $\begin{array}{l}\text { HbAlc (NGSP, } \\
\text { H) }\end{array}$ & 5.9 & 6.0 & 5.1 & 4.6 \\
\hline $\begin{array}{l}\text { Fasting serum C- } \\
\text { peptide }(\mathrm{ng} / \mathrm{mL})\end{array}$ & 0.31 & 0.72 & 0.50 & 0.73 \\
\hline $\begin{array}{c}\text { Fasting serum } \\
\text { insulin }(\mu \mathrm{IU} / \mathrm{mL})\end{array}$ & 8.99 & 4.74 & 4.09 & 2.65 \\
\hline Treatment plan & $\begin{array}{l}\text { Insulin glargine injection (10 units at bedtime), saxagliptin } \\
(2.5 \mathrm{mg} \text { once/day), voglibose ( } 0.2 \mathrm{mg} \text { three times/day) }\end{array}$ & $\begin{array}{c}\text { Metformin hydrochloride } \\
1000 \mathrm{mg} / \text { day; gliclazide } \\
160 \mathrm{mg} / \text { day }\end{array}$ & - & - \\
\hline
\end{tabular}

$\dagger$ DKA: diabetic ketoacidosis. $¥$ This individual had no diabetes.

of the luciferase assays demonstrated that $K L F 11$ plays a role in and the transcriptional regulation of the insulin gene. The insulin promoter activity induced by the KLF11 (c.1061G $>$ T) variant was observed to be lower than that induced by WT-KLF11 (Figure 3(c)). Moreover, the insulin gene activation was observed to be affected in the mutant KLF11. To further explore the role of the KLF11 Cys354Phe variant on $\beta$-cell function, we overexpressed the WT and variant KLF11 in INS1 cells (using the pcDNA3.1 plasmid as the negative control and the KLF11 (WT and Cys354Phe) plasmids) and found that the KLF11 Cys354Phe variant decreased insulin transcription and reduced insulin secretion even after stimulation with high glucose (Figures 3(d) and $3(\mathrm{e}))$.

3.6. Exploration of KLF11 (c.1061G>T) Gene Variant Treatment. The blood glucose level of the proband was under control after treatment with insulin and oral hypoglycemic agents for one and a half years (Table 2). Due to the inconvenience of using insulin at school, the medication was adjusted to oral gliclazide sustained-release tablets ( $30 \mathrm{mg} /$ day) combined with diet control in June 2020. We found that together with the control of staple food intake, this treatment resulted in better blood sugar control in a two-week staple diet trial. There was no significant difference in energy intake between the two weeks ( $p<0.05$, Table 3$)$. However, there were signif- icant differences in average daily blood glucose, fasting blood glucose, and $2 \mathrm{~h}$ postmeal blood glucose between the two weeks ( $p<0.05$, Table 3$)$. There was no statistical difference in the mean amplitude of glycemic excursions (MAGE) between the two weeks, which represented blood glucose fluctuations. According to the dynamic blood glucose parameters, dietary intervention may be beneficial for the proband and help control daily glucose levels (Figures 4(a) and 4(b)).

\section{Discussion}

The diagnosis of MODY is challenging due to its relatively low prevalence and the overlap in presentation and clinical features between patients with MODY and those with other diabetes subtypes [13]. MODY is characterized by autosomal-dominant inheritance with a multigenerational family history of diabetes, onset before 25 years of age, and the absence of pancreatic autoimmunity and insulin resistance [14]. In this study, we identified a KLF11 variant in three individuals belonging to one family via whole-exome sequencing. Among them, two individuals (including one young adult) developed diabetes. The proband exhibited hyperglycemia at 23 years of age and was observed to be negative for islet cell autoantibodies. The maternal grandfather developed type 2 diabetes at the age of 66 . The mother was considered non-diabetic,but had delayed insulin secretion 


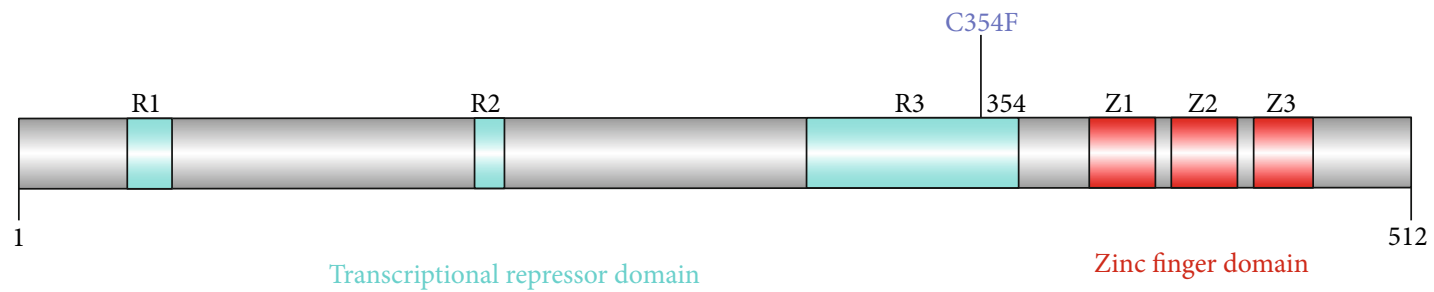

(a)

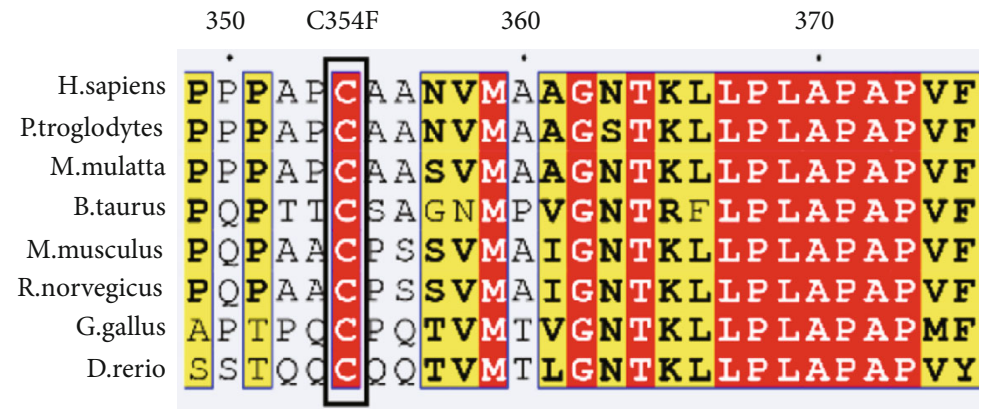

(b)
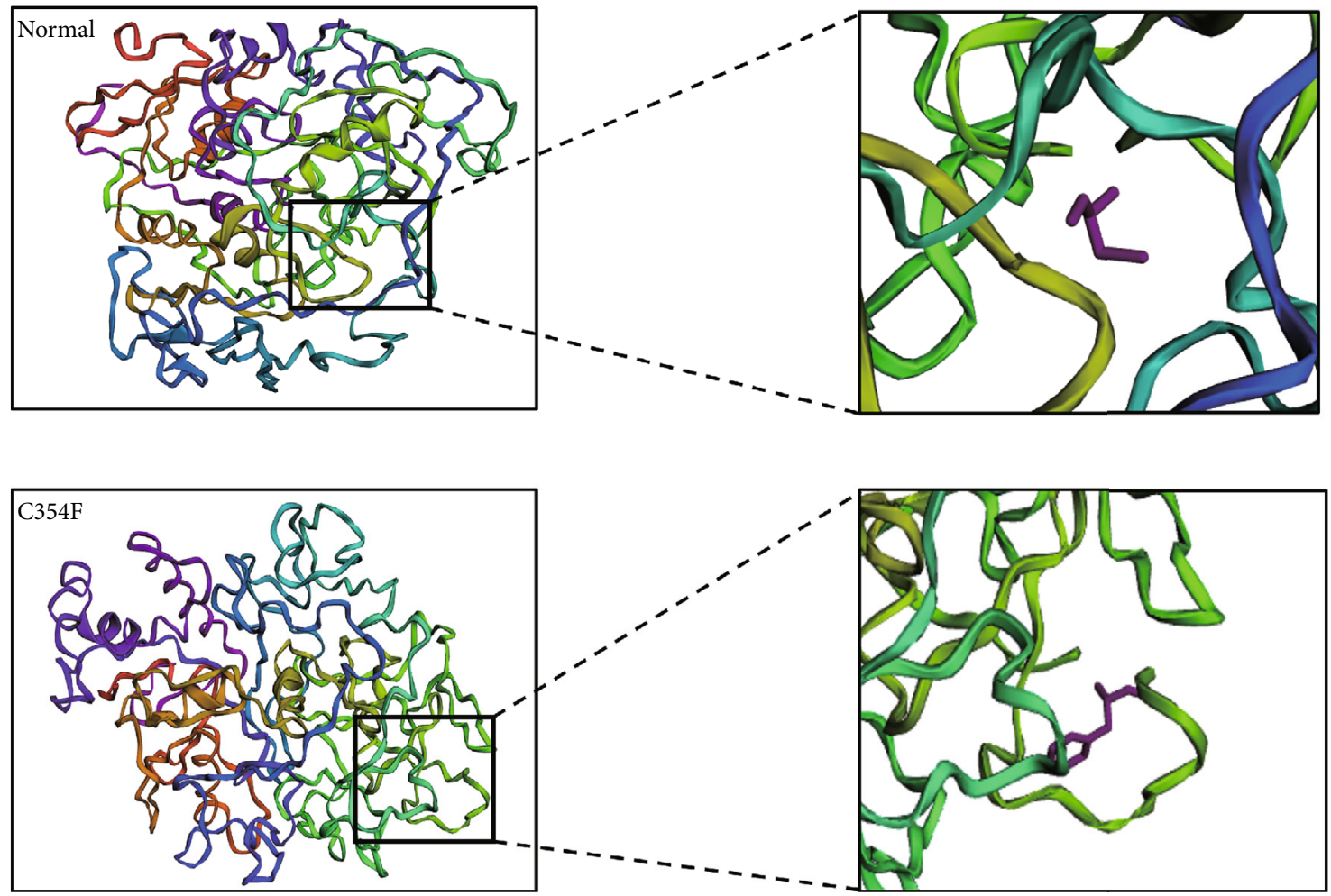

(c)

FIgURE 2: Sequencing analysis and 3D structure of the mutant protein. (a) KLF11 structure domains. Mutations at the protein level are indicated below the 3RDB domain. (b) Cross-species conservation of Cys354Phe-KLF11. (c) Protein structure prediction of KLF11 (WT and Cys354Phe).

via OGTT and islet function test, which is one of the characteristics of diabetes. The father was considered healthy. In fact, strict adherence to these guidelines confers high specificity but low sensitivity in identifying MODY subjects, as more than half of patients with confirmed mutations identified in European countries do not meet these clinical criteria for referral. As such, adherence to current guidelines will continue to lead to the misdiagnosis of a large proportion of patients with MODY $[15,16]$. Furthermore, by extending MODY diagnostic testing beyond current guidelines, Owen et al. [17] identified MODY subjects with clinical features that are not characteristic of MODY, including the absence 


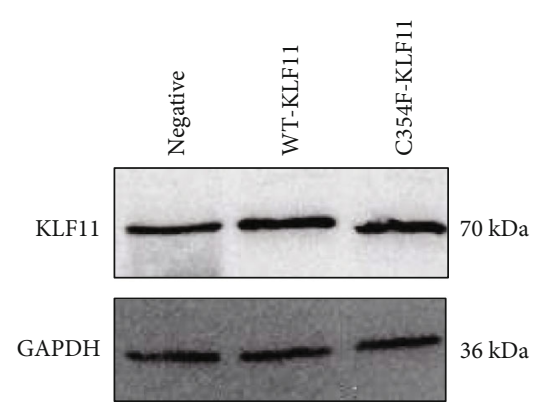

(a)

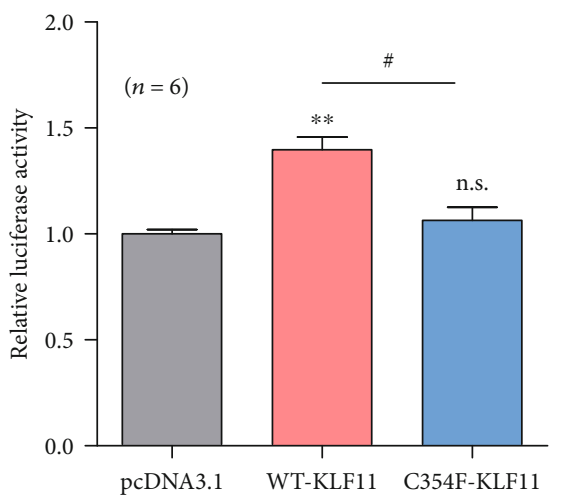

(c)

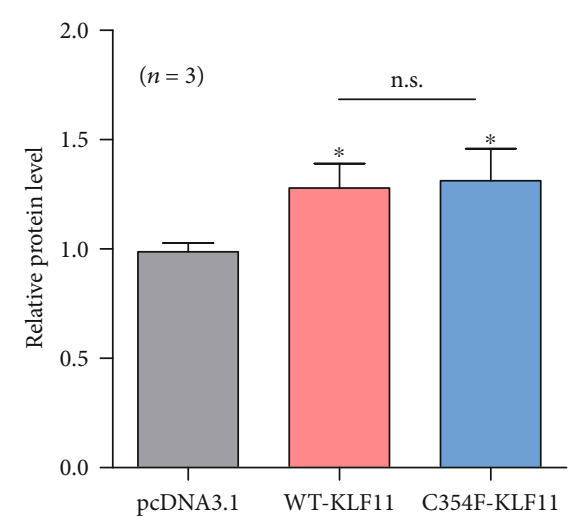

(b)

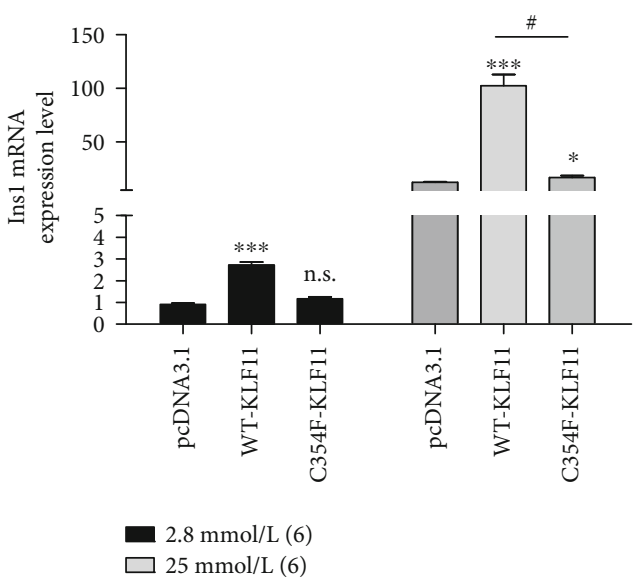

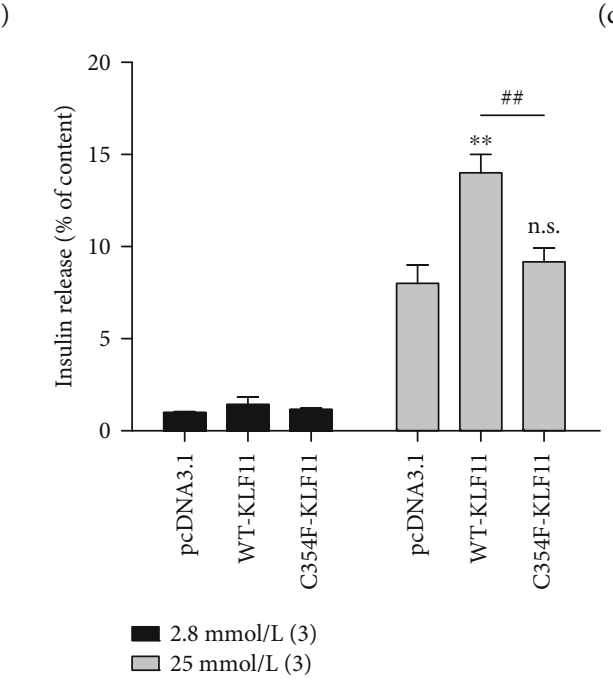

(e)

FIgure 3: Functional analysis of the KLF11 Cys354Phe variant. (a) Protein expression of KLF11 (WT and Cys354Phe). Cell lysates of KLF11expressing cells were used. Protein production was tested via western blotting. (b) Histogram of the KLF11 protein expression level analysis. (c) Luciferase assays of HEK 293 cells transfected with each KLF11 expression vector (WT and Cys354Phe). ${ }^{* *}$ denotes $p<0.01$ ( $\left.p=0.0031\right)$ for the KLF11 WT plasmid compared to the empty plasmid; n.s. denotes $p>0.05(p=0.3899)$ for KFL11/C354F compared to the empty plasmid; \# denotes $p<0.05(p=0.0176)$ for KFL11/C354F compared to KLF11 WT. (d-f) INS1 cells were transfected with the KLF11-WT or KLF11Cys354Phe plasmids for 24 (d) or $48 \mathrm{~h}$ (e). (d) qRT-PCR was conducted to determine the Ins1 mRNA levels after stimulation with $2.8 \mathrm{mmol} / \mathrm{L}$ glucose (low) or $20 \mathrm{mmol} / \mathrm{L}$ glucose (high) for $24 \mathrm{~h}$. (e) Insulin secretion levels in INS1 cells were analyzed via glucose-stimulated insulin secretion assay using ELISA after stimulation with $2.8 \mathrm{mmol} / \mathrm{L}$ glucose (low) or $20 \mathrm{mmol} / \mathrm{L}$ glucose (high) for $2 \mathrm{~h}$. Note: $n$ denotes the number of experiments. ${ }^{* *}$ denotes $p<0.01(p=0.0018)$ for KLF11 WT compared to the empty plasmid; n.s. denotes $p>0.05(p=0.1836)$ for KFL11/C354F compared to the empty plasmid; \#\# denotes $p<0.01(p=0.0027)$ for KFL11/C354F compared to KLF11 WT. 
TABLE 3: The mean and standard deviation of blood glucose and daily energy intake of the proband between the two weeks.

\begin{tabular}{|c|c|c|c|c|c|c|}
\hline \multirow[t]{2}{*}{ Variable } & & \multicolumn{2}{|c|}{$\begin{array}{c}\text { First week with staple } \\
\text { foods }\end{array}$} & \multicolumn{2}{|c|}{$\begin{array}{l}\text { Second week without } \\
\text { staple foods }\end{array}$} & \multirow[t]{2}{*}{$p$ value } \\
\hline & & Mean & SD & Mean & $\mathrm{SD}$ & \\
\hline \multirow{5}{*}{ Blood glucose $(\mathrm{mmol} / \mathrm{L})$} & Daily average & 5.65 & 0.49 & 4.16 & 0.54 & 0.000 \\
\hline & Fasting & 5.21 & 0.52 & 4.09 & 0.78 & 0.01 \\
\hline & $2 \mathrm{~h}$ after breakfast & 7.03 & 0.38 & 5.15 & 1.14 & 0.004 \\
\hline & $2 \mathrm{~h}$ after lunch & 5.8 & 1.13 & 4.2 & 1.20 & 0.054 \\
\hline & $2 \mathrm{~h}$ after dinner & 7.12 & 1.91 & 4.87 & 0.86 & 0.021 \\
\hline \multicolumn{2}{|c|}{ Mean amplitude of glycemic excursions (MAGE) } & 2.88 & 0.83 & 2.93 & 0.87 & 0.918 \\
\hline \multicolumn{2}{|c|}{ Daily energy intake (kilocalories) } & 918.28 & 287.90 & 1023.00 & 507.61 & 0.643 \\
\hline
\end{tabular}

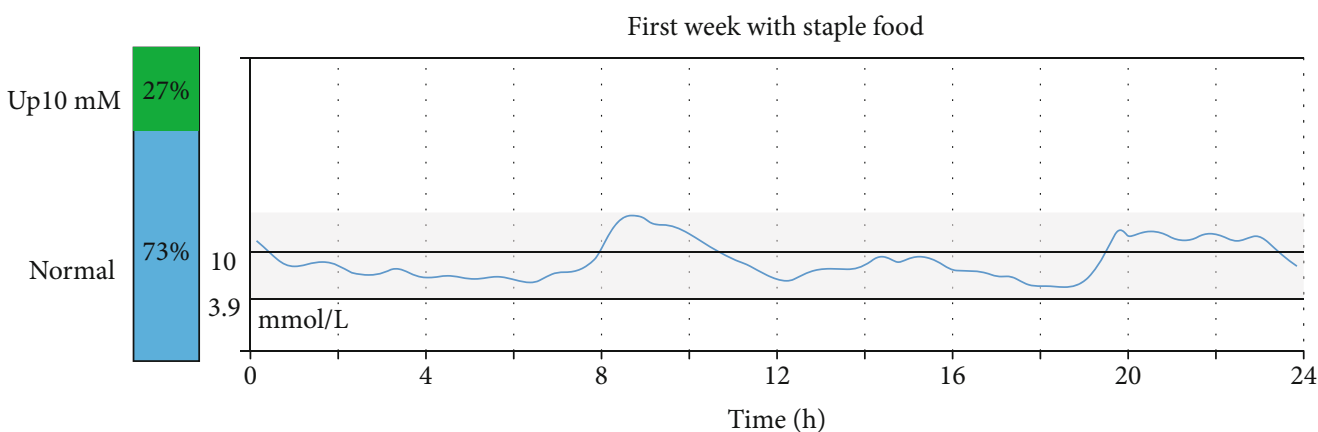

(a)

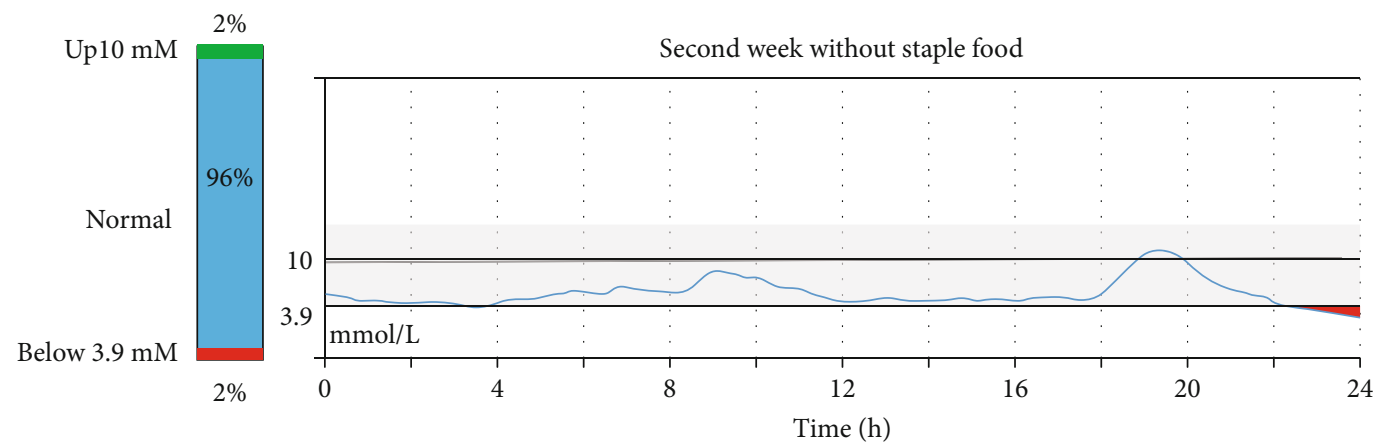

(b)

FIGURE 4: Representative data of the scanning dynamic glucose monitoring report for the proband with and without staple food intake. The proband wore the Freestyle Libre H (Abbott Diabetes Care Ltd.) for the dynamic monitoring of the blood glucose levels for 14 days. The proband needed to maintain normal staple food intake during the first week, while no staple food intake was allowed during the second week; the amount of calories for each meal was recorded through the "mint app" (China) on the phone. No exercise adjustments were performed in these two weeks. After 14 days, the data were analyzed to obtain the following results: (a) representative data of the scanning dynamic glucose monitoring report for the proband with staple food intake. The proband was shown to have a glucose level above $10 \mathrm{mmol} / \mathrm{L}$ for approximately $27 \%$ of the day; (b) representative data of the scanning dynamic glucose monitoring report for the proband without staple food intake. The proband was shown to have glucose levels above $10 \mathrm{mmol} / \mathrm{L}$ for only about $2 \%$ of the day.

of a family history of diabetes. Therefore, the diagnosis of MODY for the proband is definite, even without a typical family history.

It is well known that diabetes is the result of a combination of genetic and environmental risk factors. Among them, epigenetics plays a vital role in mediating the interaction between environmental and genetic factors. Epigenetics also has an intergenerational effect, that is, some extragene information from the grandparents will be passed to the grandchildren, and marks will be burned on the grandchildren, who show the corresponding characteristics. These factors may contribute to the earlier onset of diabetes.

Genetic studies have found that missense mutations in the KLF11 gene lead to the development of late-onset diabetes [7]. This is due to the fact that the mutations affect KLF11-binding promoters and activation of the bladder protein gene. Moreover, after mutations in the cis-acting element of KLF11 were found to inhibit KLF11-induced activation of the insulin gene, leading to a decrease in the biosynthesis of insulin in the body. The KLF11 protein is a zinc 
finger transcription factor that binds to SP1-like sequences in the promoter region of the $\varepsilon$ - and $\gamma$-globin genes [18]. Three transcript variants encoding two different isoforms have been found for KLF11 mRNA, and their proteins are expressed in pancreatic $\beta$-cells [10]. This binding increases its repression and impairs the activation of insulin promoters [19]. In addition, defects in this gene cause MODY7. Furthermore, Neve et al. identified two rare KLF11 variants (Ala347Ser and Thr220Met) in families with early onset type 2 diabetes, which were shown to significantly impair the transcriptional activity of KLF11. Furthermore, they discovered a frequent polymorphic Gln62Arg variant that was significantly associated with type 2 diabetes mellitus in North European populations [2]. Ushijima et al. identified a heterozygous KLF11 (p.His418Gln) variant in a family that was clinically diagnosed with early childhood-onset type $1 \mathrm{~B}$ diabetes [6]. These two diabetes-associated studies revealed the effects of a loss of KLF11 protein function.

Multiple amino acid sequence alignments showed that KLF11 Cys354Phe (C354F) was conserved across various species. Moreover, the cells transfected with the KLF11-WT and mutant plasmid constructs exhibited no statistically significant differences (data not shown). Moreover, there were no significant differences in the protein expression levels between the KLF11-WT- and KLF11-C354F-transfected cells. Notably, the KLF11 (c.1061G $>$ T) variant induced lower insulin promoter activity than KLF11-WT. This indicates that the mutant affects the KLF11-induced activation of the insulin gene. It is predicted that mutations at this buried site may lead to its exposure on the surface of the protein, thereby altering the protein activity $[20,21]$. In addition, due to the exposure of this site at the surface of the protein after mutation, it might be possible that the site is located in a larger transcriptional blocking domain, thereby affecting the transcriptional functions of insulin [22]. Further cell function studies showed that the KLF11 Cys354Phe variant decreased insulin transcription and reduced insulin secretion even upon stimulation with high glucose. Moreover, dysregulation of KLF11 also affected the insulin content of cells.

Children and adolescents diagnosed with diabetes may initially be treated with insulin, and this regimen often continues even after the stabilization of glycemia. However, in some patients with MODY, hyperglycemia can be controlled by prescribing oral antidiabetic drugs (e.g., sulfonylureas), without the use of insulin [23]. In fact, standard treatments have not been established for most diabetes subtypes due to the low number of cases and lack of data confirming treatment efficacy. Administration of insulin therapy from the initial phase of MODY7 has been described in previous study [6], which is consistent with the treatment received by the proband in our study. In this case, using insulin (using and storage defects) also led to inconvenience for the proband. Moreover, after treatment with sulfonylureas and when the total energy intake remained stable by reducing the intake of staple foods, we found that the proband's blood glucose levels were better controlled. Therefore, oral antidiabetic drugs and dietary intervention may benefit diabetic patients with KLF11 mutations and help them control their daily glucose levels. The following regimen and a healthy lifestyle are very important factors in the disease control for patients with type 2 diabetes.

\section{Conclusions}

In summary, in this study, we successfully identified the KLF11 (c.1061G > T) variant via whole-exome sequencing, which was shown to cause MODY7 in a 23-year-old female. This study is the first to demonstrate that KLF11 (c.1061G $>$ T) variants are involved in the pathogenesis of MODY7. Epigenetic factors may contribute to the earlier onset of diabetes. Moreover, we showed that the administration of oral antidiabetic drugs and dietary interventions were beneficial for the proband and helped control the daily glucose levels. Our study also has a few limitations. Further studies, such as animal experiments, are needed to explain the association between altered KLF11 function and the diabetes pathogenesis and severity. In addition, new dietary intervention and treatment methods need to be developed for MODY.

\section{Data Availability}

The data used to support the findings of this study are available from the corresponding author upon request.

\section{Conflicts of Interest}

The authors declare that they have no conflict of interest.

\section{Authors' Contributions}

$\mathrm{XGH}$ and LC are involved with the study design. CW, RXZ, and JW are involved in the study conduct. Original draft preparation is collected by SYJ, QJR, and JW. SYJ and QJR drafted and revised the manuscript. Yujing Sun and Jingru $\mathrm{Qu}$ contributed equally to this work.

\section{Acknowledgments}

This work was supported by the Graduate Education Innovation Program of Cheeloo College of Medicine, Shandong University (Grant No. 2020Y25 to L.C.), the National Natural Science Foundation of China (Grant No. 81670706 to L.C., Grant No. 81770818 to X.G.H., Grant No. 81873632 to L.C., Grant No. 82070800 to L.C., Grant No. 81770818 to L.C.), the National Key R\&D Program of China (Grant No. 2016YFC0901204 to L.C., Grant No. 2018YFC1311801 to L.C.), and the Taishan Scholars Project (Grant No. ts201712089 to L.C.).

\section{References}

[1] M. E. Fernandez-Zapico, G. A. Lomberk, S. Tsuji et al., "A functional family-wide screening of SP/KLF proteins identifies a subset of suppressors of KRAS-mediated cell growth," The Biochemical Journal, vol. 435, no. 2, pp. 529-537, 2011.

[2] B. Neve, M. E. Fernandez-Zapico, V. Ashkenazi-Katalan et al., "From the cover: role of transcription factor KLF11 and its diabetes-associated gene variants in pancreatic beta cell 
function," Proceedings of the National Academy of Sciences, vol. 102, no. 13, pp. 4807-4812, 2005.

[3] H. Zhang, Q. Chen, M. Yang et al., "Mouse KLF11 regulates hepatic lipid metabolism," Journal of Hepatology, vol. 58, no. 4, pp. 763-770, 2013.

[4] H. Zhang, Q. Chen, T. Jiao et al., "Involvement of KLF11 in hepatic glucose metabolism in mice via suppressing of PEPCK-C expression," PLoS One, vol. 9, no. 2, article e89552, 2014.

[5] A. Loft, I. Forss, M. S. Siersbæk et al., "Browning of human adipocytes requires KLF11 and reprogramming of PPAR $\gamma$ superenhancers," Genes \& Development, vol. 29, no. 1, pp. 7-22, 2015.

[6] K. Ushijima, S. Narumi, T. Ogata et al., "KLF11 variant in a family clinically diagnosed with early childhood-onset type 1B diabetes," Pediatric Diabetes, vol. 20, no. 6, pp. 712-719, 2019.

[7] P. Firdous, K. Nissar, S. Ali et al., "Genetic testing of maturityonset diabetes of the young current status and future perspectives," Frontiers in Endocrinology, vol. 9, 2018.

[8] L. A. Kelley, S. Mezulis, C. M. Yates, M. N. Wass, and M. J. E. Sternberg, "The Phyre2 web portal for protein modeling, prediction and analysis," Nature Protocols, vol. 10, no. 6, pp. 845-858, 2015.

[9] D. L. Zhang, Y. J. Sun, M. L. Ma et al., "Gq activity- and $\beta$ arrestin-1 scaffolding-mediated ADGRG2/CFTR coupling are required for male fertility," eLife, vol. 7, 2018.

[10] G. Lomberk, A. Grzenda, A. Mathison et al., "Krüppel-like factor 11 regulates the expression of metabolic genes via an evolutionarily conserved protein interaction domain functionally disrupted in maturity onset diabetes of the young," The Journal of Biological Chemistry, vol. 288, no. 24, pp. 1774517758, 2013.

[11] H. Ishihara, T. Asano, K. Tsukuda et al., "Pancreatic beta cell line MIN6 exhibits characteristics of glucose metabolism and glucose-stimulated insulin secretion similar to those of normal islets," Diabetologia, vol. 36, no. 11, pp. 1139-1145, 1993.

[12] F. Zhang, D. Ma, W. Zhao et al., "Obesity-induced overexpression of _miR-802_ impairs insulin transcription and secretion," Nature Communications, vol. 11, no. 1, p. 1822, 2020.

[13] G. Thanabalasingham and K. R. Owen, "Diagnosis and management of maturity onset diabetes of the young (MODY)," $B M J$, vol. 343, no. oct19 3, p. d6044, 2011.

[14] F. K. Kavvoura and K. R. Owen, "Maturity onset diabetes of the young: clinical characteristics, diagnosis and management," Pediatric Endocrinology Reviews, vol. 10, no. 2, pp. 234-242, 2012.

[15] B. M. Shields, S. Hicks, M. H. Shepherd, K. Colclough, A. T. Hattersley, and S. Ellard, "Maturity-onset diabetes of the young (MODY): how many cases are we missing?," Diabetologia, vol. 53, no. 12, pp. 2504-2508, 2010.

[16] C. Bellanné-Chantelot, D. J. Lévy, C. Carette et al., "Clinical characteristics and diagnostic criteria of maturity-onset diabetes of the young (MODY) due to molecular anomalies of the HNF1A gene," The Journal of clinical endocrinology metabolism, vol. 96, no. 8, pp. E1346-E1351, 2011.

[17] G. Thanabalasingham, A. Pal, M. P. Selwood et al., "Systematic assessment of etiology in adults with a clinical diagnosis of young-onset type 2 diabetes is a successful strategy for identifying maturity-onset diabetes of the young," Diabetes Care, vol. 35, no. 6, pp. 1206-1212, 2012.
[18] Y. Atlasi, W. Megchelenbrink, T. Peng et al., "Epigenetic modulation of a hardwired 3D chromatin landscape in two naive states of pluripotency," Nature Cell Biology, vol. 21, no. 5, pp. 568-578, 2019.

[19] A. Mathison, A. Grzenda, G. Lomberk et al., "Role for Krüppel-like transcription factor 11 in mesenchymal cell function and fibrosis," PLoS One, vol. 8, no. 9, article e75311, 2013.

[20] G. S. Daftary, Y. Zheng, Z. M. Tabbaa et al., "A novel role of the $\mathrm{Sp} / \mathrm{KLF}$ transcription factor KLF11 in arresting progression of endometriosis," PLoS One, vol. 8, no. 3, article e60165, 2013.

[21] A. Broom, Z. Jacobi, K. Trainor, and E. M. Meiering, "Computational tools help improve protein stability but with a solubility tradeoff," The Journal of Biological Chemistry, vol. 292, no. 35, pp. 14349-14361, 2017.

[22] C. R. Knoverek, G. K. Amarasinghe, and G. R. Bowman, "Advanced methods for accessing protein shape-shifting present new therapeutic opportunities," Trends in Biochemical Sciences, vol. 44, no. 4, pp. 351-364, 2019.

[23] T. Urakami, "Maturity-onset diabetes of the young (MODY): current perspectives on diagnosis and treatment," Diabetes, Metabolic Syndrome and Obesity: Targets and Therapy, vol. Volume 12, pp. 1047-1056, 2019. 